\title{
COMUNICACIÓN Y AFRONTAMIENTO COMO ESTRATEGIAS INDIVIDUALES QUE BUSCAN FACILITAR LA ADAPTACIÓN SOCIAL EN POBLACIÓN MIGRANTE
}

\author{
ANGÉLICA OJEDA GARCÍA, * JOSÉ CUENCA VÁZQUEZ, ** \\ DYANA IVETTE ESPINOSA GARDUÑO***
}

RESUMEN: La migración es considerada como un fenómeno psicosociocultural que influye en diversas esferas del individuo que emigra. En su intento por alcanzar un nivel de adaptación social óptimo, los migrantes y sus familias se enfrentan a una serie de cambios y estresores asociados, viéndose en la necesidad de desarrollar ciertas habilidades cognitivas e instrumentales. El presente artículo tuvo como objetivo conocer los distintos estilos de afrontamiento que utiliza el migrante en respuesta a los cambios por los que atraviesa y en qué forma se focaliza por establecer, fomentar y fortalecer sus redes sociales de apoyo, utilizando la comunicación como medio. La muestra estuvo constituida por 178 inmigrantes mexicanos en Estados Unidos. Al mismo tiempo los datos presentes, sugieren algunos indicadores que favorecen el mantener, fomentar y multiplicar las redes sociales de apoyo, con el objeto de potencializar sus recursos para alcanzar un nivel de adaptación social óptimo.

palabras ClaVE: Adaptación, Migración, Comunicación, Afrontamiento, Apoyo.

ABSTRACT: Migration is a psycho-socio-cultural phenomenon that impacts different aspects of an individual's life. Migrants and their families must face a variety of changes and stressors while attempting to reach an optimal level of social adaptation. This necessitates the development of certain cognitive and instrumental skills. This article examines the different styles of coping used my migrants and the ways in which they establish, foster, and strengthen social support networks by using communication as a tool. Our sample comprised 178 Mexican immigrants in the United States. At the same time, the data suggests that there are some indicators that favor the preservation, fostering and multiplication of social support networks, as these can serve as resources that help them reach an optimal level of social adaptation.

KEYwORDs: Adjustment, Migration, Communication, Confrontation, Support.

*Profesora e investigadora de tiempo completo de la Universidad Iberoamericana, en el Departamento de Psicología, angelica.ojeda@uia.mx

**Licenciado en Psicología, en la Universidad Iberoamericana con dirección anterior, jocuen@ yahoo.com.mx

***Estudiante del último semestre de la Licenciatura en Psicología, en la Universidad Iberoamericana con dirección anterior, diespinosa@hotmail.com 
L

a migración es considerada como un fenómeno psicosociocultural, pues genera cambios importantes en todas las esferas del individuo (tanto a nivel social, de inserción y estabilidad a la comunidad, individual, fami-

liar, económica, etc.) y sus acciones como resultado del desarraigo y el estrés asociado con el momento de partir, el encuentro con la nueva cultura y estilo de vida. Por lo que a los inmigrantes se les ha considerado como un grupo de alto riesgo, en el desarrollo de alteraciones psicológicas y repercusiones a nivel de salud mental (Berry, 1992).

No obstante, el proceso de migración afecta, en forma paralela, tanto a los individuos que emigran como a las familias que dejan en su lugar de origen. Fenómeno que, como se va dando paulatina y lentamente, exige cierto nivel de adaptación social; al cuál dentro de la literatura del tema, se le refiere bajo el término de «ajuste del migrante» (Bhugra, 2004).

En ese sentido, para alcanzar dicho ajuste óptimo, el migrante requiere desarrollar, por un lado, una adaptación emocional/afectiva, que se refleja en el nivel de bienestar subjetivo y satisfacción al nuevo estilo de vida que adopta y que resulta de las nuevas circunstancias, el manejo y el afrontamiento que se hace de situaciones del día a día. Mientras que por otro lado, una adaptación sociocultural y conductual, la cual se puede observar a través del aprendizaje de habilidades y conocimientos culturalmente apropiados, del lugar de destino. Entre tales habilidades se pueden considerar: mantenimiento de cierto contacto de apertura, intercambio, retroalimentación y con ello, enfrentamiento a los problemas de la vida cotidiana provenientes del exterior, pero desde la óptica del contexto del que ahora forman parte (Ataca y Berry, 1998).

De acuerdo con Ataca y Berry (1998) para que se logre una buena adaptación psicológica, todo depende del tipo de personalidad del individuo, del manejo y ajuste que ha hecho de los cambios por los que ha atravesado en su historia y del apoyo o redes sociales con las que cuente. Mientras que la adaptación sociocultural depende del conocimiento que se tenga acerca de la nueva cultura, del distanciamiento cultural, de la identidad cultural, de la habilidad en el manejo del lenguaje de la sociedad receptiva, tiempo de residencia dentro de la nueva cultura, y cantidad de contacto con los miembros de la sociedad dominante. A esta adaptación, Torres y Rollock (2004) le dan el nombre de «aprendizaje cultural».

Tanto la adaptación psicológica como la adaptación sociocultural se encuentran interrelacionadas. De alguna manera, cualquiera o las dos, permiten al migrante hacer un mejor manejo de las situaciones inesperadas y el estrés constante de cambio al que se enfrenta. Ambas, en combinación, promueven interacciones positivas y la vulnerabilidad necesaria que favorece el aprendizaje cultural en los migrantes, con los miembros de la sociedad receptora. Cabe mencionar al mismo tiempo que, ambas: la adaptación psicológica y sociocultural pueden ser conside- 
radas como dos herramientas indispensables para fomentar un adecuado manejo de los conflictos por «choque cultural» que pueden repercutir tanto en su bienestar psicológico, en la planeación de un proyecto de vida, planteamiento de metas a corto, mediano y largo plazo; así como en debilitar, fortalecer o incrementar sus redes de apoyo.

Para conocer, precisamente, el nivel de aprendizaje cultural de un individuo migrante, es necesario visualizar su grado de dominio de habilidades instrumentales y funcionales, para desempeñarse socialmente con autoeficiencia, en ciertas tareas culturales, dentro de contextos específicos (LaFramboise, Coleman y Gerton, 1993; Ogbu, 1981).

\section{ESTRATEGIAS DE AFRONTAMIENTO: UN MEDIO PARA RESPONDER A LOS CAMBIOS Y AL PROCESO MIGRATORIO}

Lazarus (1982) define el afrontamiento como parte de los recursos con los que cuenta una persona y que se expresan a través de todos aquellos esfuerzos cognitivos y conductuales que se desarrollan para responder, disminuir, reducir, minimizar, controlar, dominar, tolerar, o solucionar los problemas en situaciones estresantes y emociones negativas, provenientes del exterior (el ambiente) o del interior (ambiente familiar) y el contexto sociocultural (Góngora, 2000) e implica el manejo y ajuste a dichos cambios (Lazarus y Folkman, 1984).

Las habilidades, estrategias o esfuerzos cognitivos pueden a su vez organizarse en dos categorías, LaFramboise et al. (1993) y Ogbu (1981):

a) Habilidades de afrontamiento individual. Que involucran habilidades típicamente preescritas por la cultura principal (la que recibe al inmigrante). El afrontamiento individual refiere a una configuración de características personales o procesos internos que los individuos utilizan para involucrarse en relaciones persona-ambiente, a través de demandas particulares. Éste, en cuanto a la forma de un abordaje orientado al dominio y solución de problemas de los eventos de la vida, se ha asociado a altos niveles de funcionamiento, a través de varios grupos étnicos americanos (Tyler, Brome, y Williams, 1991).

b) Habilidades de afrontamiento general (de respuesta cultural). Habilidades específicas de grupo que se originan en la cultura tradicional (de donde proviene el inmigrante) y que son determinadas como necesarias para el éxito en el nuevo ambiente (Sodowsky y Lai, 1997). Las minorías étnicas desarrollan sus propias teorías y estrategias específicas de grupo para lidiar con el nuevo ambiente; éstas se fundan y son características de la cultura tradicional y sus experiencias (Hobfoll, 1998; Ogbu, 1981).

Dentro de las habilidades de afrontamiento individual, Lazarus y Folkman (1984/1991) describen cuatro modos básicos de afrontamiento: 1) Estrategias ins- 
trumentales o Confrontación o Acción directa. Son aquellas dirigidas hacia el manejo de la amenaza o el estresor mismo, en forma directa y precisa a través de hacer cosas para solucionarlo, implementar nuevas estrategias de solución, actuar, no quedarse con los brazos cruzados. 2) Estrategias intrapsíquicas. Son aquellas dirigidas principalmente a regular o minimizar el conflicto emocional acompañante, a través de minimizar el valor que se le da a la emoción negativa y buscar autoafirmación de las emociones positivas, es decir, buscar cambiar el sentimiento y el pensamiento con respecto a lo que el evento genera en la persona. 3) Inhibición de la acción Escape-evitación. Son aquellas dirigidas a evitar confrontar el evento estresor y sustituirlo por alguna otra acción que haga que se le olvide el primero, con el objeto de que sea el tiempo el que acomode, por sí mismo, las cosas. 4) Búsqueda de información o Solución Planeada: Son aquellas dirigidas a buscar cualquier medio instrumental para planear su solución, por ejemplo pedir un consejo, movilizar apoyo emocional entre las redes sociales con las que se cuenta, buscar paz interior hablando o rezándole a Dios para luego poder pensar con más calma, escribir los pros y contras del evento; así como las posibilidades de acción y la viabilidad de las mismas.

Dentro de las habilidades de afrontamiento general y respuesta cultural, en población migrante, Bouchard (2003) refiere que de acuerdo a su naturaleza y razón de ser, las estrategias o estilos de afrontamiento pueden ser clasificadas en: estrategias enfocadas al problema, o bien, a la emoción. Donde las primeras implican una «planeación» que conduzca a la solución del problema (Parker y Endler, 1996) y las segundas, se refieren a todas aquellas actividades enfocadas a modificar las reacciones emocionales e internas resultantes de la situación estresante, conocida como distanciamiento/evitación (Chang, 1998).

Para decidir entre si «planean» o «evitan» el conflicto, primero deben hacer una valoración primaria que se refiere al impacto y significancia que el evento tiene sobre la persona y que responde a la pregunta: ies la situación una amenaza para mi bienestar? Y una valoración secundaria, que tiene que ver con discernir entre todos los recursos con los que cuenta la persona para lidiar con la situación estresante y que responde a la pregunta: itengo las habilidades para hacer frente a la situación-problema? En otras palabras, si debo aprender a manejarla, y qué tanto me siento con el nivel de competencia, en cuanto a habilidades y conocimientos para responder a ella.

Lazarus y Folkman (1984) consideran que, para comprender la relación entre los esfuerzos individuales de afrontamiento y la salud mental, independientemente del efecto que genere dicho afrontamiento, de que se solucione o no el conflicto emocional, todo afrontamiento repercute en el bienestar de la persona, disminuye (es decir, beneficia de algún modo y en algún grado a la persona) sin importar la naturaleza o nivel de estrés del problema que se enfrenta, moderando el impacto aversivo de todo estresor. 
McCrae (1991) enfatiza que, aunque la forma de enfrentar o afrontar las adversidades depende de las características culturales, como es el caso de los migrantes y su intento por lograr una adaptación socio-cultural y conductual, en esencia todos comparten las siguientes características:

a) La mayoría de los esfuerzos de afrontamiento se conciben como respuestas a estresores externos, como: enfermedades, divorcio, duelo e internos.

b) Estas respuestas a dichos estresores, se consideran conscientes.

c) Al afrontar un problema, se propicia el cambio, como respuesta a que se tiene identificado el origen del problema.

Bouchard (2003) en su estudio con migrantes encuentra que las personas que emigran, y además manifiestan bajos recursos emocionales (lo que equivale a conflicto emocional), recurren a hacer una valoración primaria del evento y a utilizar más estrategias enfocadas en la emoción y, con ello buscan auto-modificarse para que no les impacte tanto la situación amenazante del proceso migratorio (Terry, 1994). Por el contrario, quienes tienden a analizar el problema desde diferentes perspectivas, es decir, no nada más a partir de lo que sienten o perciben, sino a partir del hecho o el contenido del problema mismo, comúnmente, hacen una valoración secundaria de éste y por ello, logran hacer una planeación de la estrategia encaminada a su solución (Costa, Somerfield y McCrae, 1996).

Algunos autores coinciden (p.e.: Aldwin y Revenson, 1987; Lazarus y Folkman, 1984/1991; Pearlin y Schooler, 1978) que lo que las personas hacen o dejan de hacer al tratar con sus problemas puede ser la diferencia para su bienestar, pues lo importante no es que el problema al que se enfrenta sea de índole catastrófica, imprevista, incontrolable, vital, o bien, sólo una pequeña contrariedad, lo esencial es el cómo los perciben y qué hacen ante esos problemas.

\section{ESTUDIOS SOBRE MIGRACIÓN}

Giunta y Compas (1993) realizaron un estudio para conocer el estilo de afrontamiento más utilizado entre los miembros de parejas casadas migrantes, en cuanto al manejo de una situación estresante. Ellos evaluaron a 153 parejas de diferentes partes que residían en el Noreste de Vermont (EUA), con un nivel socioeconómico medio. Les aplicaron la Escala de Estilos de Afrontamiento de Folkman y Lazarus (1985). Sus resultados muestran que el estilo de afrontamiento utilizado se asocia con algunos síntomas psicológicos. Además, los afrontamientos de los maridos y esposas están asociados uno con el otro. En ese sentido, el estilo de afrontamiento de escape-evitación (aquél que evita afrontar el problema y prefiere hacer otras cosas, con tal de no pensar en él) utilizado con mayor frecuencia por las esposas está 
asociado con síntomas psicológicos de los esposos; mientras que el afrontamiento de los maridos no está asociado con síntomas psicológicos de las esposas.

Aldwin y Revenson (1987) realizaron un estudio longitudinal sobre estrés por aculturación y salud mental, donde evaluaron a 291 adultos migrantes (provenientes de diferentes países) residentes en Los Ángeles, California y encontraron evidencia sobre un ciclo causal reforzado entre pobre salud mental y estrategias de afrontamiento desadaptativas. Mientras más grande sea el nivel inicial de conflicto emocional y el grado de severidad del problema, es más probable que los individuos utilicen un afrontamiento desadaptativo, incrementando así el conflicto emocional y probablemente incrementando también la posibilidad de problemas en el futuro. En cuanto al afrontamiento enfocado en el problema, es insuficiente inquirir sobre las estrategias utilizadas, se requiere más información sobre los resultados de estas estrategias (Felton y Revenson, 1984).

Finalmente, se ha visto aunque no exactamente en población migrante, que pese a pertenecer a la misma cultura, se encuentran diferencias entre hombres y mujeres en el estilo de afrontamiento. Por ejemplo, algunos estudios realizados en México con jóvenes han encontrado que, a mayor cercanía del individuo con la problemática, más respuestas afectivas, dato congruente con las culturas colectivistas, donde la familia y las relaciones cercanas con los otros juegan un papel importante. Góngora y Reyes, (1998) reportan que, aunque tanto hombres como mujeres dan respuestas directas e instrumentales, las mujeres usan más el enfrentamiento emocional, datos que también reportan Harada, Alfaro y Villagrán (2002) con jóvenes mexicanos.

\section{DEL AFRONTAMIENTO A LA ACULTURACIÓN: LA COMUNICACIÓN COMO PUENTE}

Un vez que el migrante ha logrado afrontar los cambios que la inserción a la nueva cultura implica, el proceso migratorio pasa de ser sólo un proceso de adaptaciones y ajustes a la adopción de una forma de responder predominante al contexto. A esta forma de responder, dentro de la literatura se le conoce como «aculturación» y no es otra cosa que una adaptación cultural.

Un migrante puede aculturarse: a) Asimilándose totalmente a la nueva cultura y olvidándose de su cultura de origen. b) Separándose totalmente de la nueva cultura, conservando y practicando las costumbres de la cultura de origen. c) Integrándose a través de prácticas de ciertas costumbres de la cultura destino y manteniendo algunas o todas las tradiciones de la cultura de origen. En particular, la situación que realmente no indica aculturación y que refleja mayor conflicto psicológico y choque cultural entre lo que marca la cultura de origen y la destino es: marginalizarse a través de no practicar las tradiciones y costumbres de la cultura destino, pero al mismo tiempo dejar de practicar y conservar las festividades del lugar de origen (Berry, Poortinga, Segall y Pierre, 1999). 
Es así como la aculturación puede ser entendida como un proceso de expansión y manejo del nivel de adaptación tanto psicológica como sociocultural, visto como un producto concreto en conductas como: fluidez del lenguaje, elección de la comida, preferencias en cuanto a encuentros sociales, celebración de festividades, valores culturales observados, manejo del idioma (Berry et al., 1999), por lo que algunos autores también la han nombrado «aculturación psicológica» (Graves, 1967).

Lin, Tazuma y Masuda (1979) han encontrado que quienes emigran de sociedades socio-céntricas a culturas egocéntricas, como es el caso de los mexicanos que emigran a los Estados Unidos, pueden presentar dificultad para ajustarse a la sociedad recién llegada, debido a que esta última espera que se comporten de cierta manera, diferente a la que ellos están acostumbrados. Los cuatro tipos de organización familiar en los migrantes que se evaluaron fueron: familia nuclear, familia extendida, mujeres divorciadas o solteras y hombres solteros; ellos observaron que las mujeres solas fueron las que menos contaban con recursos personales para lograr un buen ajuste social, pues su estabilidad emocional tendía a la depresión; mientras que los hombres solteros fueron los que reflejaron mayor energía, disposición y actitud para ajustarse a la nueva cultura.

Se ha visto que para enfrentar la vulnerabilidad social, los migrantes se apoyan en las redes sociales, ya que le ofrecen al migrante un sentido de pertenencia y significado social, ayudándolo, por tanto, a localizarse y ubicarse en el nuevo lugar (Weiss, 1989; cita en Bhugra, 2004).

Con la finalidad de promover la cercanía entre los integrantes de una relación - dividir las tareas que le dan mantenimiento al hogar, de compartir valores, de darle continuidad a su identidad, interactuar e intercambiar significados, cosas, sentimientos, ideas y formas de interpretar la vida (Sátir, 1986)-con sus paisanos, los migrantes encuentran en la familia y las relaciones significativas de su red de inserción en el lugar receptor, información estratégica sobre el modo acerca del cómo responder a las restricciones impuestas por la estructura de oportunidades en el que se encuentran inmersos y que muchas veces desconocen, oportunidades laborales, acerca de cómo mejorar su calidad de vida y mejorar su infraestructura física, construir medios de defensa y apoyo de migrantes en Estados Unidos (Ariza, 2002).

De tal forma que para que un individuo inmigrante logre una buena adaptación y funcionamiento social en el lugar destino, misma que le repercutirá en el nuevo estilo de vida que construye, es necesario que su organización familiar cambie a la par de las circunstancias. Cambios que deben ser asimilados de tal manera que formen parte de la filosofía familiar y generen congruencia entre la identidad individual y social de cada miembro y su familia.

La comunicación es el medio simbólico para incrementary mantener las redes sociales, cuyo estudio y análisis resulta de gran importancia para conocer el nivel de adaptación social del inmigrante en el nuevo entorno (Sátir, 1986). 
Por su parte Sánchez Aragón y Díaz Loving (2003), hacen una revisión exhaustiva acerca de cuáles son los estilos de comunicación que los adultos utilizan dentro de sus relaciones, y observan una tendencia a que dentro de la literatura, la mayoría de los autores tienden a hablar de estilos de comunicación "positivos» como aquellos que permiten fortalecer el vínculo, desarrollo personal y/o crecimiento como pareja y "negativos», refiriéndose a aquellos donde persiste un interés muy personal, poca apertura a escuchar, atender las necesidades y escuchar al otro. Dentro de los primeros y en lo que respecta a fomentar las relaciones cercanas y redes de apoyo, son dos los estilos de comunicación que suelen expresarse: la Comunicación íntima que es aquella que busca fortalecer el diálogo subjetivo y fomentar el vínculo emocional y, la Comunicación funcional que es aquella que se establece como una forma de organizar y distribuir todo lo referente a las tareas del hogar, del cuidado, atención y educación de los hijos.

Se ha visto que la pareja inmigrante mantiene una comunicación más de tipo funcional, es decir, sus miembros se comunican sólo y exclusivamente para la distribución y organización de funciones maritales-hogareñas (Fitzpatrick, 1988; Ojeda, 2006), dejando de lado la comunicación íntima.

En concreto, la pregunta a responder en la presente investigación fue: ¿Cuáles son las estrategias que favorecen la adaptación social y funcional de los migrantes mexicanos en los Estados Unidos? Para responder, se pensó que primero habría que conocer cuáles son las estrategias de afrontamiento que expresan los migrantes mexicanos en Estados Unidos y cuáles los estilos de comunicación que utiliza dicho grupo. En esa misma línea, cuáles en conjunto favorecen una tendencia a alimentar, mantener, incrementar o expandir sus redes sociales de apoyo y con ello, facilitar su proceso de adaptación social y aculturación a la nueva cultura.

MÉTODO

\section{Participantes}

La muestra estuvo conformada por 178 mexicanos casados migrantes de México hacia los Estados Unidos, 124 fueron mujeres (56.4\%) y 96 hombres (43.6\%), procedentes de: Distrito Federal, 36.7\%; Jalisco, 15.5\%; Michoacán, 9.7\%; Zacatecas, 5.8\%; Guanajuato, 4\%; Guerrero, 3.5\% y Puebla, 3.5\%. El rango de edad iba de 18 a 60 años, con una media de 36.5 y una desviación estándar de 10.8 años. El rango de edad de casados iba de 1 a 38 con una media de 13.4 y una desviación estándar de 9.7. El $84.3 \%$ de la muestra dijo vivir actualmente con su pareja. El rango de estudios iba de: sin estudios formales hasta posgrado incompleto, con un promedio 
de secundaria incompleta y una desviación estándar hacia por debajo de primaria incompleta y una hacia arriba de preparatoria o vocacional incompleta. La selección de la muestra fue accidental no probabilística.

\section{Instrumentos}

Inventario de Estilos de Comunicación de Nina Estrella (1991) en versión corta, adaptada para este estudio y cuyas $\alpha$ de Cronbach, se refieren a las obtenidas por un estudio piloto previo al estudio que se presenta en este artículo. La adaptación de dicho instrumento, se hizo como sigue: de los 36 adjetivos que lo componen, un estudio piloto previo con la población objeto mostró que algunos de los adjetivos que constituyen la escala no eran discriminativos y se eliminaron durante dicho procedimiento; de tal suerte que la escala de aplicación, finalmente quedó constituida por 17 características en formato de Diferencial Semántico con cinco espacios y en versión pictórico. Aunque la versión original la constituyen dos subescalas, una dirigida al estilo del cónyuge que responde y la otra, al estilo de la pareja desde el punto de vista del que responde (con confiabilidad de $\alpha=.78$ y $\alpha=.91$, respectivamente), se decidió dejar una sola subescala, que es reflejo de una autodescripción y ofrece el punto de vista del respondiente en primera persona. Por combinación de los adjetivos, el estilo resultante puede ir desde: Comunicador Positivo $(\alpha=.8808)$, Comunicador Negativo ( $\alpha=$.8982), Comunicador Reservado-reflexivo $(\alpha=.7997)$ hasta expresarse como Comunicador Violento $(\alpha=.7355)$.

Escala Multidimensional y Multisituacional de Estilos de Afrontamiento en versión corta, es decir de los cinco ambientes que evalúa se tomaron sólo las áreas de ambiente familiar y situaciones laborales, como una forma de observar, cotidianamente, el estilo de afrontamiento que los migrantes reportaron ante problemas en estas áreas (Góngora \& Reyes, 1998). Es una escala multidimensional y multisituacional que mide una dimensión duradera y estable (afrontamiento como rasgo) y una segunda dimensión, cambiante y temporal (afrontamiento como estado) con seis situaciones problema: vida (situación general), salud, familiares, pareja, amigos, escuela y/o trabajo (situaciones más específicas). Cada situación consta de 18 reactivos, haciendo un total de 108, con un formato tipo Likert pictográfico con un continuo de siete puntos que van de siempre (7) a nunca (1). De la cual sólo se 
tomó un grupo de 18 reactivos y se aplicó en la muestra objeto bajo la situación problemática de: eventos estresantes o problemas a solucionar durante su proceso como migrante. La aplicación de la escala de afrontamiento que aquí se reporta, fungió tanto como piloteo de la misma como de diagnóstico para la muestra objeto.

\section{Procedimiento}

La recolección de la muestra se hizo a través de algunos contactos de instituciones con migrantes en los Estados Unidos, a través de los consulados móviles, los consulados mexicanos en Estados Unidos, quienes nos introdujeron en las zonas de: San Bernardino, Palm Desert, Bloomington, Riverside, Thermal, Coachella Valley, Mecca, Indio, Palm Springs y en Duros. A los participantes se les pidió firmar un consentimiento voluntario, porque así lo exige la ley estadounidense y en caso de alguna auditoria, asegurándoles su confidencialidad. La aplicación de los instrumentos se hizo tanto en forma individual como grupal. En caso de requerirlo, se les leían las preguntas y el entrevistador iba anotando las respuestas dadas por el entrevistado.

\section{RESULTADOS}

Como las escalas utilizadas no fueron hechas en población migrante, sino adaptadas en lo que respecta a la redacción de sus reactivos, se decidió iniciar por conocer cuál fue el comportamiento de los reactivos para la muestra objeto. Del análisis de Frecuencias y Jicuadrada por escala, se desecharon aquellos reactivos que no discriminaron entre la población estudiada. Posteriormente, de los reactivos que sí discriminaron, se sometieron a un Análisis Factorial de Componente Principales con rotación varimax y valores eigen mayores a 1 . Los resultados permitieron observar dos estilos tanto de promover la comunicación como dos estrategias de afrontamiento a los problemas (tabla 1). En el primer caso, los factores explican el $63.34 \%$ y en el segundo, el $61.35 \%$ de la varianza. Posteriormente, para conocer la consistencia interna, tales factores fueron sometidos a análisis de confiabilidad a través del Alpha de Cronbach (tabla 1). 
TABLA 1

Factores Resultantes de cada uno de los Inventarios aplicados para la muestra estudiada

\begin{tabular}{|c|c|c|c|c|c|}
\hline \multicolumn{6}{|c|}{ INVENTARIO DE ESTILOS DE COMUNICACIÓN DE NINA ESTRELLA (1991) EN VERSIÓN CORTA, ADAPTADA PARA ESTE ESTUDIO } \\
\hline F1= COMUNICACIÓN NEGATIVA & $\mathrm{N}=8$ & $X=1.8 .2$ & $S=.007$ & $\alpha=.8973$ & PESO FACTORIAL \\
\hline \multicolumn{5}{|l|}{ Dominante } & .824 \\
\hline \multicolumn{5}{|l|}{ Conflictivo/a } & .767 \\
\hline \multicolumn{5}{|l|}{ Confuso/a } & .766 \\
\hline \multicolumn{5}{|l|}{ Evasivo/a } & .755 \\
\hline \multicolumn{5}{|l|}{$\mathrm{Seco} / \mathrm{a}$} & .733 \\
\hline \multicolumn{5}{|l|}{ Agresivo/a } & .719 \\
\hline \multicolumn{5}{|l|}{ Gritón/a } & .648 \\
\hline \multicolumn{5}{|l|}{ Callado/a } & .627 \\
\hline F2 = COMUNICACIÓN POSITIVA & $\mathrm{N}=3$ & $X=2.2$ & $S=.006$ & $\alpha=.8045$ & PESO FACTORIAL \\
\hline \multicolumn{5}{|l|}{ Conciliador/a (nada conciliador) } & .858 \\
\hline \multicolumn{5}{|l|}{ Afectuoso/a (nada afectuoso) } & .817 \\
\hline \multicolumn{5}{|l|}{ Respetuoso/a (nada respetuoso) } & .745 \\
\hline \multicolumn{6}{|c|}{ ESCALA MULTIDIMENSIONAL Y MULTISITUACIONAL DE ESTILOS DE ENFRENTAMIENTO (GÓNGORA \& REYES, 1998) EN VERSIÓN CORTA } \\
\hline F1= AFRONTAMIENTO ACTIVO & $\mathrm{N}=20$ & $X=12.02$ & $S=4.5$ & $\alpha=.964$ & PESO FACTORIAL \\
\hline \multicolumn{5}{|c|}{ Aunque no los resuelva del todo, los intento solucionar } & .931 \\
\hline \multicolumn{5}{|c|}{ Planeo como solucionarlo y lo llevo a acabo } & .927 \\
\hline \multicolumn{5}{|c|}{ Los analizo para solucionarlos } & .925 \\
\hline \multicolumn{5}{|c|}{ Trato de aprender de ellos, encontrándoles lo positivo } & .903 \\
\hline \multicolumn{5}{|c|}{ Sé lo que debo hacer y pongo todo mi empeño para que las cosas salgan bien } & .893 \\
\hline \multicolumn{5}{|c|}{ Trato de utilizar nuevas estrategias para resolverlos } & .891 \\
\hline \multicolumn{5}{|c|}{ Busco las opciones que tengo para solucionarlos } & .886 \\
\hline \multicolumn{5}{|c|}{ Reflexiono acerca de lo que puedo hacer o decir } & .870 \\
\hline \multicolumn{5}{|c|}{ Tengo fe, en que se solucionará en algún momento } & .815 \\
\hline \multicolumn{5}{|c|}{ Me preocupo } & .803 \\
\hline \multicolumn{5}{|c|}{ Pienso que son pruebas que nos pone la vida } & .753 \\
\hline \multicolumn{5}{|c|}{ Deseo que la situación termine o desaparezca } & .753 \\
\hline \multicolumn{5}{|c|}{ Me prometo que la próxima vez no me vuelve a pasar } & .737 \\
\hline Hablo con alguien para aclarar & leas & & & & .732 \\
\hline Me imagino cómo pueden salir & sas & & & & .720 \\
\hline Asumo la responsabilidad de lo & ucede & trario de & o a otr & & .712 \\
\hline $\begin{array}{l}\text { Me tranquilizo para no actuar } \\
\text { que pienso }\end{array}$ & sivame & y dejarn & var por & rimero & .678 \\
\hline Le platico a alguien sobre ellos, & ando $u$ & onsejo & & & .644 \\
\hline Rezo & & & & & .548 \\
\hline Busco la comprensión de los de & & & & & .418 \\
\hline F2 = AFRONTAMIENTO PASIVO & $N=4$ & $X=2.2$ & $S=.002$ & $\alpha=.6885$ & PESO FACTORIAL \\
\hline Me imagino que no estoy pasa & or eso y & e estoye & a mejor & ación & .762 \\
\hline Me olvido de ellos, haciendo cc & ferente & & & & .740 \\
\hline Dejo que el tiempo lo resuelva & & & & & .736 \\
\hline Hago como si nada estuviera $p$ & & & & & .666 \\
\hline
\end{tabular}

Nota: Los reactivos con carga factorial negativa, se leen al contrario de lo que indican, para que se incluyan como parte de determinado factor. 


\section{Donde}

Comunicación Negativa. Es el tipo de comunicación que se da con la finalidad de dominar la información que se maneja dentro de la relación, donde no se da la apertura ni se busca el intercambio de ideas, es decir, se da en forma seca, con poco diálogo, un tanto conflictiva, nada afectuosa, en forma agresiva y evasivamente.

Comunicación Positiva. Es el tipo de comunicación que busca sobre todo conciliar con el otro miembro de la pareja, de una manera afectiva y respetuosa.

Afrontamiento Activo. Es la estrategia de resolución de problemas que implica acción, planeación, reflexión sobre lo sucedido y las consecuencias del evento, pedir un consejo, platicarle a alguien más el problema como una forma de aclararse las ideas, implementar nuevas formas de abordarlo hasta preocuparse y rezar.

Afrontamiento Pasivo. Es la estrategia para responder a los problemas de la vida cotidiana de manera inactiva y dejándolos a la deriva, en espera de que sea el tiempo quien los resuelva, o imaginando que nada esta pasando.

Una vez integrados los factores o estilos de comunicación y afrontamiento, el siguiente paso fue ver con qué frecuencia éstos se presentan, pues ello nos podría llevar a conocer cuál es la tendencia en la forma de responder a los problemas cotidianos, y qué tanto favorecen el vínculo emocional entre las personas que les son significativas y cercanas. Para ello, la gráfica 1 muestra la distribución de los factores que se obtuvieron de las escalas aplicadas, en la muestra estudiada.

En el caso de la Escala de Estilos de Comunicación, se observa que fue el estilo Negativo el que se da con mayor frecuencia entre la población estudiada (55.70\%); mientras que entre los estilos de Afrontamiento, fue el Activo, el más predominante (59\%). Lo que permite inferir por un lado, poco interés entre los migrantes evaluados, por atender y alimentar afectivamente las relaciones cercanas; mientras que por otro lado, una atención considerable en responder de la manera más adecuada, planeada, exitosa y asertiva a los problemas con los que se van enfrentando, en su intento por alcanzar una adaptación funcional.

Para poder ver en qué forma se interrelaciona la manera de fomentar las redes sociales cercanas, con el interés por resolver, en toda la extensión de la palabra, activamente sus problemas, se procedió a realizar una correlación Producto-Momento de Pearson entre los distintos factores resultantes en este estudio. La tabla 2 permite ver que entre dichas correlaciones, sólo se observa una correlación interescalar, positiva, alta y significativa entre el factor de comunicación Positiva y el estilo de Afrontamiento Activo. En otras palabras, dicho resultado refiere que cuando se da entre los miembros de la relación de pareja la conciliación de ideas y acuerdos, de una manera afectuosa y respetuosa, es más probable que se confronten las situaciones estresantes de la vida cotidiana, activamente: buscando alternativas para su solución, confrontándolos, aprendiendo de ellos, responsabilizándose de los mismos, o bien, de manera inactiva, pero haciendo algo como es: platicando en espera de un consejo externo, preocupándose o rezando por ellos. 


\section{GRÁFICA 1}

Distribución por Estilos de Comunicación y Afrontamiento

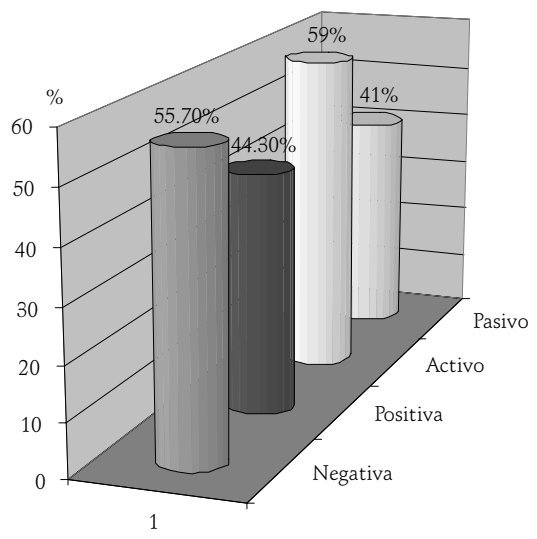

De forma contraria y tristemente, en este caso los migrantes evaluados reportaron manifestar con mayor frecuencia y en relación a su pareja, un estilo de comunicación negativo, lo que necesariamente choca negativa y significativamente con los estilos más constructivos y de adaptación, el estilo de comunicación positivo $\left(-.389^{* *}\right)$ y el estilo de afrontamiento activo $\left(-.319^{* *}\right)$. Y positivamente, con el estilo de afrontamiento pasivo $\left(.308^{* *}\right)$ de confrontación de situaciones cotidianas.

TABLA 2

Correlaciones Momento-Producto de Pearson entre los factores que resultaron del Inventario de Estilos de Comunicación y la Escala Multidimensional y Multisituacional de Estilos de Afrontamiento, en versión corta para este estudio.

\begin{tabular}{|l|c|c|c|}
\hline \multicolumn{1}{|c|}{ FACTORES } & NEGATIVO & POSITIVA & ACTIVO \\
\hline Negativo & - & & \\
\hline Positiva & $-.389^{* *}$ & - & \\
\hline Activo & $-.319^{* *}$ & $.707^{* *}$ & - \\
\hline Pasivo & $.308^{* *}$ & - & $.257^{* *}$ \\
\hline
\end{tabular}

Nota: ${ }^{*} \mathrm{p}=.001,{ }^{*} \mathrm{p}=.01$

\section{Discusión}

Los resultados de este estudio permitieron observar, tal como la literatura lo refiere, que existe una tendencia de ajuste constante por parte del migrante, en pro de alcanzar una pronta y efectiva adaptación social (Bhugra, 2004), por lo que se recurre a una diversidad de estrategias de afrontamiento, según la tarea a resol- 
ver y el contexto en el que se den (LaFramboise et al., 1993; Ogbu, 1981). En este estudio se vio que, cuando de afrontar problemas cotidianos se trata, la estrategia de afrontamiento activo fue la más utilizada; mientras que cuando de fomentar, mantener, ampliar y multiplicar redes sociales se trata, la estrategia de comunicación más utilizada es la negativa, es decir, aquella donde se evita el intercambio de ideas, más bien, se busca dominar la información que se maneja dentro de la relación, en forma seca, con poco diálogo, un tanto conflictiva, nada afectuosa, en forma agresiva y evasiva. Con estos datos, parece que a los migrantes estudiados no les interesa alimentar mucho el vínculo emocional y significativo de las posibles redes sociales que pueda formar, lo que lleva a pensar que su foco de interés en maximizarlas sería con el objeto de utilizarlas como estrategias de tipo instrumental, como lo refieren otros estudios (p.e.: LaFramboise et al., 1993; Parker y Endler, 1996; e informativo, que facilite el afrontamiento de estresores externos (Ariza, 2002; Lazarus y Folkman, 1984/1991) como es la tendencia cuando de resolución de problemas se trata (McCrae, 1991). No obstante, algunos autores refieren que para lograr una efectiva y eficiente funcionalidad social, primero se debe trabajar la adaptación emocional (p.e.: Ataca y Berry, 1998; Bouchard, 2003).

Por lo que en la lucha por afrontar los estresores asociados al proceso migratorio y alcanzar un nivel de adaptación social óptimo (Ataca y Berry, 1998), independientemente de si ésta puede ser direccionada hacia lo emocional, psicológico o sociocultural, los datos de este estudio permiten ver una tendencia a probar una gran variedad de estrategias de resolución de problemas y apertura hacia el aprendizaje cultural (Torres y Rollock, 2004), tales como: intentar todo lo que sea posible por resolver el conflicto, implementar nuevas estrategias, aprender del problema buscando lo positivo, reflexionar y analizar, responsabilizarse de las consecuencias hasta buscar la comprensión de los demás. Pues, el hecho de que el estilo de afrontamiento activo haya resultado como la estrategia más utilizada entre los migrantes mexicanos en Estados Unidos, coincide con las estrategias de afrontamiento de tipo instrumentales y de habilidad general (LaFramboise et al., 1993; Sodowsky y Lai, 1997). Donde todo esfuerzo es una muestra de confrontación del problema directo, por eso se le considera ya como algo beneficioso, aminora el conflicto emocional y amortigua el estrés, simplemente por la atención que se le pone al mismo (Lazarus y Folkman, 1984/1991), ya que lo más importante es responder, no evadir o evitar (Pearlin y Schooler, 1978).

A pesar de que en este estudio, las distintas opciones que se le presentaron a los evaluados, en cuanto a diferentes formas de responder a un conflicto, el análisis factorial las agrupó como parte del factor de afrontamiento activo, lo cual resulta un poco contradictorio con lo que refieren Lazarus y Folkman (1984/1991) acerca de que cualquier tipo de estrategia de afrontamiento favorece la adaptación social a la nueva cultura, siempre y cuando ésta sea congruente y genere armonía entre dicho esfuerzo cognitivo elegido para la resolución del conflicto y la salud mental que éste le genera a la persona; con ello, la presencia de una gran diversidad 
de opciones para poder resolver un conflicto, en la mayoría de las veces produce que la efectividad de los mismos disminuya.

Haciendo un análisis exhaustivo y cualitativo por ítems que integran la estrategia de afrontamiento activa, se pudo observar que las formas de responder más utilizadas, en primer lugar son aquellas que buscan en una forma directa abordar el problema (hacer lo que está en sus manos para responder y resolverlo), seguido por los reactivos directo-revalorativo, es decir, pensar, analizar o reflexionar sobre las alternativas de abordaje y finalmente, la forma activa que va desde hacer cosas, pedir un consejo, preocuparse o rezar. En otras palabras, el intento por probar nuevas posibilidades, abre una gama de sucesos en función de hacerse la vida ligera, de vivir la experiencia en forma más tranquila, reconfortante y constructiva, en un intento de lograr «aculturarse» hacia el nuevo lugar (Berry et al., 1999).

De tal suerte que la interacción con otras variables puede ser señal de salud mental. Los resultados de este estudio ofrecen que el diálogo se funda más en la acción que en el intercambio de ideas y pensamientos, pues el estilo de comunicación más expresado entre los migrantes estudiados fue el Negativo (Sánchez Aragón y Díaz-Loving, 2003), como una forma de disminuir la importancia de alimentar el vínculo emocional, y más bien promover la comunicación con aquellos cercanos, que funjan como red social estratégica informativa. En este sentido, al correlacionar los estilos de afrontamiento con los estilos de comunicación expresados por la muestra estudiada, pudimos ver una tendencia, el estilo positivo de afrontamiento (estilo activo) con el de comunicación positiva (estilo positivo) y viceversa, el estilo negativo de afrontamiento (estilo pasivo) con el de comunicación negativa (estilo negativo). Situación que suena congruente con la tendencia de todo aquel migrante que busca adaptarse, funcionar socialmente, construir redes sociales reales y efectivas.

\section{BIBLIOGRAFÍA}

AlDwin, C.M. y T.A. Revenson (1987), «Does coping help? A reexamination of the relation between coping and mental health", Journal of Personality and Social Psychology, 53(2): 337-348.

ArizA, M. (2002), «Migración, familia y transnacionalidad en el contexto de la globalización: algunos puntos de reflexión», Revista Mexicana de Sociología, vol. 64, núm. 4, octubre-diciembre, pp. 53-84.

ATACA, B. y John W. Berry (1998), "Psychological, sociocultural and marital adaptation of Turkish immigrant couples in Canada» [Versión electrónica], International Journal of Psychology, 37: 13-26.

BerRY, John W. (1992), «Acculturation and Adaptation in a new society», Migration, 30: 69-86. , Ype H. Poortinga, H.M. Segall y R.D. Pierre (1999), Cross-cultural psychology. Research and Applications, United of America, Cambridge University Press.

Bhugra, Dinesh K. (2004), "Migration and mental health», Acta Psychiatry Scandinavia, 109: 243-258. 
BOUCHARD, Guylaine (2003), «Cognitive appraisals, neuroticism, and openness as correlates of coping strategies: an integrative model of adaptation to marital difficulties», Canadian Journal of Behavioral Science, 35(1): 1-12.

CHANG, Eric C. (1998), «Dispositional optimism and primary and secondary appraisal of a stressor: Controlling for confounding influences and relations to coping and psychological and physical adjustment», Journal of Personality and Social Psychology, 74: 1109-1120.

Costa, Paul T., M.R. Somerfield y R.R. McCrae (1996), "Personality and coping», en M. Zeidnery N.S. Endler (editores), Handbook of coping; Theory, research, applications, Nueva York, John Wiley, pp. 45-61.

Cummings, E. Mark y A. Watson O'Reilly, (1997), «Fathers in family context: Effects of marital quality on child adjustment», en M.E. Lamb (editor), The role of the father in child development, Nueva York, John Wiley, 3a. ed., pp. 49-65.

FeLton, Barbara J. y Revenson, Tracey A. (1984), «Coping with chronic illness: A study of illness controllability and the influences of coping strategies on psychological adjustment», Journal of Consulting and Clinical Psychology, 52: 343-353.

FitzPATRICK, Mary Anne (1988), «Between husbands and wives, communication in relationships», en B. Rubin (editor), Communication yearbook, Nueva Bunswick.

Folkman, Susan y Richard S. Lazarus (1985), "If it changes it must be a process: Study of emotion and coping during three stages of a college examination", Journal of Personality and Social Psychology, 48: 150-170.

Giunta, Carole T. y Bruce Compas (1993), «Doping in marital dyads: Patterns and associations with psychological symptoms», Journal of Marriage and the Family, 55: 10111017.

GónGora, Elías e I. Reyes Lagunas (1998), «El enfrentamiento a los problemas en jóvenes adultos yucatecos», La Psicología Social en México, vol. 7, pp. 18-24.

(2000), El enfrentamiento a los problemas y el papel del control: Una visión etnopsicológica en ecosistema con tradición, Tesis de doctorado, no publicada, México, Universidad Nacional Autónoma de México.

Graves, Theodore D. (1967), «Psychological acculturation in a tri-ethnic community», Southwestern Journal of Anthropology, 23: 337-350.

Harada, Olivares Eduardo, Lilia Bertha Alfaro Martínez y Gabina Villagrán Vázquez (2002), "Percepción de riesgo y estrategias de enfrentamiento ante el embarazo no planeado: Diferencias entre hombres y mujeres preparatorianos", La Psicología Social en México, vol. 9, pp. 192-199.

Hobfoll, Stevan E. (1998), Stress, culture, and community: The psychology and philosofy of stress, Nueva York, Plenum Press.

LaFramboise, Teresa, L.K. Coleman Hardin y Jennifer Gerton (1993), «Psychological impact of biculturalism: Evidence and theory", Psycholgical Bulletin, 114: 395-412.

LAZARUS, Richard S. (1982), «Thoughts on the relations between emotion and cognition», American Psychologist, 37: 1019-1024.

(1984/1991), "On the primacy of cognition», American Psychologist, 39: 124-129.

(1982), "Thoughts and the relations between emotion and cognition», American Psychologist, 37: 1019-1024.

y S. Folkman (1991/1994), Estrés y procesos cognitivos, México, Ediciones Roca. 
y S. Folkman (1984), Stress, appraisal, and coping, Nueva York, Springer Publishing.

Lin, Keh Ming, Laurie Tazuma, y Minoru Masuda (1979), "Adaptational problems of Vietnamese refugees I. Health and mental health isuues», Archivo General Psychology, 36: 955-961.

Mancillas Bazan, Celia (2004), La construcción de la intimidad, la identidad y los roles de género en parejas del valle de Chalco, Tesis de doctorado, no publicada, México, Universidad Iberoamericana.

McCary, James y S. McCary (1983), Sexualidad humana, México, Manual Moderno.

McCrae, Robert (1991), "The five-factor model and its assessment in clinical settings", Journal of Personality and Social Psychology, 57: 399-414.

Miller, Sherod, Elam Nunnally y Daniel Wackman (1976), "A communication training program for couples», Social Casework, enero.

Nina Estrella, R. (1991), Comunicación marital y estilos de comunicación: Construcción y validación, Tesis de doctorado no publicada, Facultad de Psicología, Universidad Nacional Autónoma de México.

OGBu, John (1981), "Origin of human competence: A cultural-ecological perspective», Child Development, 52: 413-429.

OJEDA, Angélica (2006), «Satisfacción Marital en Parejas Migrantes: Un Análisis por Combinación de Estilos de Apego/Amor», Revista Iberoamericana de Diagnóstico y Evaluación, 1: 21, 23-45.

Parker, James D.A. y Norman S. Endler (1996), "Coping and defense: A historical overview», en M. Zeidner y N.S. Endler (editores), Handbook of coping: Theory, research, applications, Nueva York, John Wiley, pp. 3-23.

Pearlin, Leonard I. y Carmi Schooler (1978), «The structure of coping. Journal of Health and Social Behavior», 19: 2-21.

SÁnchez Aragón, Rozzana y Rolando Díaz-Loving (2003), «Patrones y estilos de comunicación en la pareja: Diseño de un inventario», Anales de Psicología, España, Servicio de publicaciones de la Universidad de Murcia, diciembre, 19, 2: 257-277.

SÁtir, Virginia (1986), Human Relationships inside Familiar Nucleo, México, Pax-México, pp. 122-139.

SoDowsky, Gargi Roysircar y E.W. Lai (1997), «Asian immigrant variables and structural models of cross-cultural distress», en A. Booth, A.C. Crouter y N. Landale (editores), Immigrant and the Family: Research and policy on U.S. immigrants, Mahwah, NJ: Erlbaum, pp. 211-234.

Sternberg, Robert J. (1986), «A triangular love theory», Psichological Review, 93: 119-135.

TerRy, D.J. (1994), «Determinants of coping: The role of stable and situational factors», Journal of Personality and Social Psychology, 66: 895-910.

Torres, Lucas y David Rollock (2004), «Acculturative distresss among Hispanics: The role of acculturative, coping, and intercultural competence» [versión electrónica], Journal of Multicultural Counseling \& Development, 32: 155-167.

TYLER, Forest B., Deborath Ridley Brome y Janice E. Williams (1991), Ethnic validity, ecology, and psychotherapy: A psychological competence model, Nueva York, Plenum Press.

TSENG, Wen-Shing T. (2001), Handbook of cultural psychiatry, San Diego, Academic Press.

Word, Beatrice L., Klebba B. Klebba y Bruce D. Miller (2000), «Envolving the bio-behavioral family model: the fit of attachment», Family Process, 39: 319-344. 